\title{
Knowledge and Perceptions of Influenza Vaccinations Among College Students in Vietnam and the United States
}

\author{
Akiko Kamimura', Ha N. Trinh"2, Shannon Weaver ${ }^{3}$, Alla Chernenko', Maziar M. Nourian ${ }^{4}$, Nushean Assasnik', Hanh Nguyen ${ }^{5}$ \\ ${ }^{1}$ Department of Sociology, University of Utah, Salt Lake City, UT; ${ }^{2}$ Center for Interdisciplinary Research in Women's Health, University of Texas \\ Medical Branch, Galveston, TX; ${ }^{3}$ Department of Health, Kinesiology and Recreation, University of Utah, Salt Lake City, UT; ${ }^{4}$ School of Medicine, \\ University of Utah, Salt Lake City, UT, USA; ${ }^{5}$ Department of Sociology, Vietnam National University, Ho Chi Minh City, Vietnam
}

Objectives: Influenza is a significant worldwide public health issue. Knowledge and perceptions regarding the flu vaccination are associated with whether individuals obtain the vaccination. The purpose of this study was to examine how such perceptions were related to knowledge and self-efficacy regarding influenza and the flu vaccination in Vietnam and the US.

Methods: College students $(n=932)$ in Vietnam $(n=495)$ and the US $(n=437)$ completed a self-administered survey regarding knowledge and perceptions of influenza vaccinations in September and October 2016.

Results: Vietnamese participants reported significantly lower levels of awareness about flu risk, higher levels of negative attitudes toward flu vaccination, lower levels of knowledge about the flu and vaccination, and lower levels of self-efficacy than US participants. Higher levels of flu and flu vaccination knowledge and self-efficacy regarding general responsible health practices were associated with lower levels of negative perceptions of flu risk and attitudes toward vaccination. At the same time, self-efficacy regarding responsible health practices was associated with higher levels of awareness of flu risk and lower levels of negative attitudes toward vaccination. Self-efficacy regarding exercise was associated with lower levels of perceptions of flu risk and higher levels of negative attitudes toward vaccination.

Conclusions: Vietnam could benefit from influenza education based on this comparison with the US. In both countries, knowledge and self-efficacy were found to be important factors influencing perceptions of influenza risk and vaccination.

Key words: Influenza, Vaccination, Beliefs, Vietnam, United States

Received: April 18, 2017 Accepted: May 17, 2017

Corresponding author: Akiko Kamimura, PhD

380 S 1530 E, Salt Lake City, UT 84112, USA

Tel: +1-801-585-5496, Fax: +1-801-585-3784

E-mail: akiko.kamimura@utah.edu

This is an Open Access article distributed under the terms of the Creative Commons Attribution Non-Commercial License (http://creativecommons.org/licenses/by$\mathrm{nc} / 4.0 / /$ which permits unrestricted non-commercial use, distribution, and reproduction in any medium, provided the original work is properly cited.

\section{INTRODUCTION}

Influenza is a significant worldwide public health issue that can cause serious health conditions, such as death in high-risk populations (e.g., children, pregnant women, the elderly, and individuals with chronic conditions), and can also negatively impact economic conditions in affected areas [1]. It is estimated that 250000 to 500000 influenza-related deaths occur worldwide annually [1]. The influenza vaccination is the most effec- 
tive way to prevent influenza or to reduce the severity of the illness [1]. However, influenza vaccination coverage is typically lower than the nationwide vaccination target goal. In the US, the target flu vaccination coverage for adults was $70 \%$, compared to the actual coverage of $41.7 \%$ in the 2015 to 2016 season [2]. The failure to reach the target vaccination goal demonstrates a pressing need to examine barriers to vaccination.

In addition to the issues of cost and accessibility, knowledge and psychological factors, such as perceptions regarding the flu vaccination, are associated with whether individuals obtain the flu vaccination [3]. The health belief model (HBM) can be used to examine vaccination self-efficacy and perceptions, as this model explains individuals' health behaviors. This model is particularly effective as it focuses on perceived susceptibility, which correlates with whether an individual obtains a vaccine. According to the HBM, knowledge is a factor that modifies individual beliefs, leading to health behavioral changes [4]. Selfefficacy refers to individual beliefs related to expectations of outcomes that may drive health behavioral changes [4]. It can be surmised that if individuals are not knowledgeable about a vaccine, they may not be motivated to discuss vaccination with their doctor. Additionally, misconceptions exist regarding the effectiveness of the flu vaccine [5].

Vietnam is a high-risk country for the spread of infectious diseases, including influenza [6]. The lack of educational campaigns seeking to raise awareness of the flu and flu vaccination remains a challenge for increasing vaccine coverage [7]. This may be due to the country's health profile and priorities. Similar to other developing countries, the prevalence of infectious diseases such as human immunodeficiency virus infection and acquired immune deficiency syndrome, tuberculosis, polio, malaria, and chickenpox remains high in Vietnam [8]. However, influenza prevention and treatment have been largely neglected across the health care system in Vietnam [9]. Previous studies on influenza in Vietnam have mainly focused on clinical testing of vaccines [10], the prevalence/epidemiology of influenza [11], or surveillance and control [12]. Little is known about perceptions, knowledge, and self-efficacy related to flu vaccinations in Vietnam. Given that perceptions, knowledge, and self-efficacy influence where and how individuals obtain flu vaccines, it is important to increase information surrounding perceptions, knowledge, and self-efficacy related to influenza vaccination in order to improve influenza vaccination coverage in Vietnam.

The purpose of this study was to examine how perceptions of influenza and flu vaccination were related to knowledge and self-efficacy in Vietnam, with a comparison group in the US. This study specifically focused on college students. Although college students are often considered a healthy population, students in the US were found to have low flu vaccination coverage and to suffer from upper respiratory health problems that negatively affected academic and work performance [13]. A literature search revealed that no studies have been conducted on knowledge and self-efficacy related to influenza vaccination among college students in Vietnam. There is also no research to date comparing self-efficacy related to influenza vaccination levels between countries. Thus, this study fills in a deficit within the current Vietnamese and US public health literature, and also provides a comparative perspective to better understand influenza-related behaviors.

\section{METHODS}

\section{Study Participants and Data Collection}

Cross-sectional data were collected using a self-administered survey in September and October 2016 at a national university in southern Vietnam and a state university in the US. Prior to data collection, this study was approved as an exempt protocol by the institutional review board (IRB) of the US university. The Vietnamese university did not have an IRB at the time of this study. The IRB of the US university has a policy regarding international research that includes cases in which there is no local IRB in another country. This study followed that policy to ensure that ethical standards of research were met both in Vietnam and in the US.

Undergraduate students 18-30 years of age were given a consent cover letter and a paper survey in classes in social science or related fields. Consent was obtained from each participant. The survey instrument was translated into Vietnamese from English for participants in Vietnam. The survey was translated by a native Vietnamese speaker who is fluent in Vietnamese and English, and was back-translated by another translator into English. The accuracy of the translation was checked by both translators. To ensure that the questions were relevant in Vietnam as well as in the US, Vietnamese researchers carefully reviewed the entire survey instrument. In Vietnam, surveys were collected in 8 classes. In the US, surveys were collected in 9 classes. The response rate was $79.5 \%$ in Vietnam and $52.7 \%$ in the US. 


\section{Measures}

\section{Perceptions of flu risk and attitudes toward vaccination}

Perceptions of flu risk and attitudes toward vaccination were measured using a scale developed by Ramsey and Marczinski [14]. The scale consists of 7 items (e.g., "The flu vaccine will not work." and "The flu vaccine is not safe.") and uses a 5-point Likert scale (1, strongly disagree; 5 , strongly agree). Scoring is based on the mean of the 7 items. Higher scores indicate higher levels of negative perceptions of flu risk and attitudes toward vaccination. The Cronbach alpha for this study population was 0.751 .

\section{Knowledge of influenza and vaccines}

Twelve statements about flu and flu vaccines (true/false/ don't know) were developed from the information about influenza provided by the Centers for Disease Control and Prevention [15]. Scoring was based on the number of correct answers to the 12 statements.

\section{Self-efficacy}

Self-efficacy was measured using the Self-Rated Abilities for Health Practices (SRAHP) scale [16]. The SRAHP uses a 5-point Likert scale ( 0 , not at all; 4 , completely) and includes 4 subscales ( 7 items each, 28 items in total): nutrition, psychological well-being, exercise, and responsible health practices. Scoring is based on a sum of the scores for the items. Higher scores indicate higher levels of self-efficacy. The Cronbach alpha values for this study population were: nutrition, 0.819 ; psychological well-being, 0.867 ; exercise, 0.918 ; and responsible health practices, 0.896 .

\section{Socio-demographic questions}

Participants were asked the following demographic questions: gender, age, year in college, major, hometown (urban or rural), whether their father had a college degree, whether their mother had a college degree, and race/ethnicity.

\section{Data Analysis}

Data were analyzed using SPSS version 22.0 (IBM Corp., Armonk, NY, USA). Descriptive data are presented as proportions for categorical variables, mean with standard deviation for continuous variables, and frequencies and percentages for categorical variables. Categorical variables were compared between countries using the chi-square test. The independentsamples $t$-test was used to compare the means of continuous

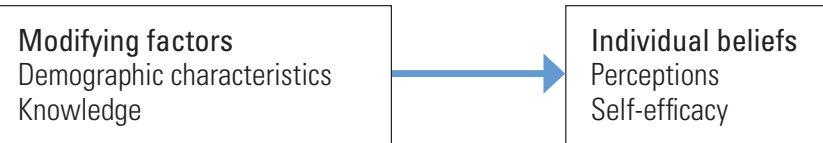

Figure 1. Application of the health belief model to this study.

variables. The HBM was chosen as an overall theoretical framework due to its emphasis on perceived susceptibility. Other models could have been chosen, such as the theory of planned behavior; however, this study focused primarily on perceptions, rather than on constructs such as motivation to comply. Figure 1 demonstrates the application of the HBM to this particular study. The hypothesis that there was a difference in perceptions of flu risk and attitudes toward vaccination depending on socio-demographic characteristics and self-efficacy was tested utilizing the factors outlined in Figure 1.

\section{RESULTS}

Table 1 presents descriptive statistics. There were 932 participants in total (495 in Vietnam and 437 in the US). Vietnamese participants reported significantly lower levels of perceptions about flu risk, higher levels of negative attitudes toward flu vaccinations, lower levels of knowledge about the flu and vaccination, and lower levels of self-efficacy in all aspects (nutrition, psychological well-being, exercise, and responsible health practices) than US participants ( $p<0.01$ ).

Table 2 presents the predictors of perceptions of flu risk and attitudes toward vaccination. Higher levels of knowledge about the flu and vaccination $(p<0.01)$ and of self-efficacy related to responsible health practices $(p<0.05)$ were significantly associated with lower levels of negative perceptions of flu risk and attitudes toward vaccination. Higher levels of selfefficacy related to exercise were significantly associated with higher levels of negative perceptions of flu risk and attitudes toward vaccination $(p<0.01)$.

\section{DISCUSSION}

This study examined perceptions, knowledge, and self-efficacy related to influenza vaccinations in Vietnam and the US. There are three main findings. First, Vietnamese participants reported significantly lower levels of awareness of flu risk, higher levels of negative perceptions and attitudes regarding flu vaccination, lower levels of knowledge about the flu and 
Table 1. Descriptive statistics

\begin{tabular}{|c|c|c|c|c|}
\hline & Total $(\mathrm{n}=932)$ & Vietnam ( $n=495)$ & US $(n=437)$ & $p$-value ${ }^{1}$ \\
\hline Women & $653(71.1)$ & $370(74.7)$ & $283(64.8)$ & $<0.01$ \\
\hline Freshman & $264(28.3)$ & $171(34.5)$ & $93(21.3)$ & $<0.01$ \\
\hline Hometown in urban area & $518(55.6)$ & $183(37.0)$ & $335(76.7)$ & $<0.01$ \\
\hline Father has college degree & $383(41.1)$ & $128(25.9)$ & $255(58.4)$ & $<0.01$ \\
\hline Mother has college degree & $340(36.5)$ & $109(22.0)$ & $231(52.9)$ & $<0.01$ \\
\hline Age & $20.19 \pm 1.99$ & $19.8 \pm 1.59$ & $20.64 \pm 2.29$ & $<0.01$ \\
\hline Perceptions of flu risks and attitudes toward vaccination & $2.45 \pm 0.56$ & $2.53 \pm 0.50$ & $2.36 \pm 0.61$ & $<0.01$ \\
\hline Knowledge of flu & $5.24 \pm 2.65$ & $4.45 \pm 2.27$ & $6.14 \pm 2.75$ & $<0.01$ \\
\hline \multicolumn{5}{|l|}{ Self-efficacy for health practices } \\
\hline Total & $76.08 \pm 18.48$ & $68.04 \pm 14.80$ & $84.45 \pm 18.23$ & $<0.01$ \\
\hline Nutrition & $20.02 \pm 4.71$ & $18.71 \pm 3.94$ & $21.49 \pm 5.05$ & $<0.01$ \\
\hline Psychological well-being & $18.97 \pm 5.04$ & $17.71 \pm 4.27$ & $20.34 \pm 5.44$ & $<0.01$ \\
\hline Exercise & $17.64 \pm 6.34$ & $15.00 \pm 5.45$ & $20.55 \pm 5.97$ & $<0.01$ \\
\hline Responsible health practices & $18.91 \pm 5.57$ & $16.59 \pm 4.72$ & $21.51 \pm 5.30$ & $<0.01$ \\
\hline
\end{tabular}

Values are presented as frequency $(\%)$ or mean \pm standard deviation.

${ }^{1} T$ The $p$-values were based on the chi-square test for categorical variables and the independent-samples $t$-tests for continuous variables compared between Vietnamese and US participants.

Table 2. Predictors of perceptions of flu risk and attitudes toward vaccination

\begin{tabular}{lcc}
\hline & Estimate & p-value \\
\hline Age & -0.02 & NS \\
Women & -0.04 & NS \\
Freshman & -0.10 & NS \\
Home town in urban area & -0.05 & NS \\
Father has college degree & -0.04 & NS \\
Mother has college degree & -0.09 & NS \\
Vietnam & 0.03 & NS \\
Knowledge of flu & -0.04 & $<0.01$ \\
Self-efficacy & & \\
$\quad$ Nutrition & -0.01 & NS \\
Psychological well-being & 0.001 & NS \\
Exercise & 0.01 & $<0.01$ \\
Responsible health practice & -0.01 & $<0.05$ \\
(constant) & 3.25 & $<0.01$ \\
R $^{2}$ & 0.10 & \\
F & 6.94 & \\
$p$-value & $<0.01$ &
\end{tabular}

NS, not significant.

vaccination, and lower levels of self-efficacy than US participants. Second, higher levels of flu and flu vaccination knowledge and of self-efficacy related to general responsible health practices were associated with lower levels of negative perceptions of flu risk and attitudes toward vaccination, regardless of country. Third, regardless of nationality, while self-effi- cacy related to responsible health practices was associated with higher levels of correct perceptions about flu risk and lower levels of negative attitudes toward vaccination, self-efficacy related to exercise was associated with lower levels of correct perceptions of flu risk and higher levels of negative attitudes toward vaccination.

These results, which indicate that Vietnamese participants had lower levels of awareness about flu risk, more negative attitudes toward vaccination, lower levels of knowledge about the flu and vaccination, and lower levels of self-efficacy related to health practices than US participants, suggest the need to promote flu vaccination education programs for college students in Vietnam. To date, research into the health of Vietnamese college students has mainly focused on substance use and mental health issues [17] or sexual behaviors [18]. Vietnamese college students could benefit from an increase in information surrounding diverse health issues, such as vaccination habits.

The results that higher levels of knowledge about the flu and vaccination and of self-efficacy related to responsible health practices were significantly associated with higher levels of awareness of flu risk and attitudes toward vaccination are consistent with the HBM. The framework drawn from the HBM to understand flu vaccination-related behaviors is applicable to individuals in Vietnam and in the US. These results may be helpful in enhancing our conceptual understanding of factors that affect flu vaccination behaviors.

Self-efficacy in relation to responsible health practices was 
associated with higher levels of perceptions of flu risk and lower levels of negative attitudes toward vaccination. Self-efficacy related to exercise was correlated with lower levels of perceived flu risk and higher levels of negative attitudes toward vaccination. Previous studies have indicated that individuals who maintain healthy lifestyles tend to believe they do not need a flu shot [19]. These kinds of beliefs can create misconceptions about the risk factors for influenza and vaccinations [19] that may cause long-term adverse health outcomes [20]. College students who believe they lead healthy lifestyles may benefit from different further education regarding flu vaccines.

This study has some limitations. It is a cross-sectional study and therefore is limited when it comes to determining causal relationships among the variables. In addition, only one university from each country was surveyed. Thus, generalizability may be limited. Furthermore, the questions measuring the levels of knowledge about the flu and flu vaccinations were developed based on US-based materials and have not been validated, and might have given some disadvantages to Vietnamese participants. Finally, it is important to be careful in comparing data from 2 different countries because influenza is sensitive to region, season, and weather, and perceptions are also associated with culture. Future studies could conduct a similar survey in Vietnam and the US and compare the data independently. For the purposes of this study, however, a dependent comparison was chosen to fill in the gap of research comparing the 2 countries. This study contributed valuable knowledge to a void in research surrounding perceptions of flu vaccinations among college students in Vietnam compared to US college students. Future research should examine health care providers' attitudes towards flu vaccines and their likelihood of recommending flu vaccines to patients.

\section{ACKNOWLEDGEMENTS}

We gratefully acknowledge the students who participated in this study and the contributions of Amanda Bertana, Wade Cole, Julia Franklin, An Ha, Shane J. Macfarlan, Theresa Martinez, Huu Binh Nguyen, Xuan Anh Nguyen, Rebecca Owen, Richard R. Paine, Tu Anh Pham, Thomas Quinn, Naveen Rathi, Maria Reyes, Mary Stoddard, and Lindsey Wright for in translation, data collection, or and data entry.

\section{CONFLICT OF INTEREST}

The authors have no conflicts of interest associated with the material presented in this paper.

\section{ORCID}

\author{
Akiko Kamimura http://orcid.org/0000-0001-6528-5770 \\ Ha N. Trinh http://orcid.org/0000-0003-2448-1016 \\ Maziar M. Nourian http://orcid.org/0000-0002-3335-9357 \\ Alla Chernenko http://orcid.org/0000-0003-3548-2698
}

\section{REFERENCES}

1. World Health Organization. Influenza (seasonal) [cited 2017 Apr 13]. Available from: http://www.who.int/mediacentre/ factsheets/fs211/en/.

2. Centers for Disease Control and Prevention. Flu vaccination coverage, United States, 2015-16 influenza season [cited 2017 Apr 13]. Available from: https://www.cdc.gov/flu/fluvaxview/ coverage-1516estimates.htm.

3. Bish A, Yardley L, Nicoll A, Michie S. Factors associated with uptake of vaccination against pandemic influenza: a systematic review. Vaccine 2011;29(38):6472-6484.

4. Champion VL, Skinner CS. The health belief model. In: Glanz K, Rimer BK, Viswanath K, Orleans CT, editors. Health behavior and health education: theory, research, and practice. 4th ed. San Francisco: Jossey-Bass; 2008, p. 45-66.

5. Centers for Disease Control and Prevention. Misconceptions about seasonal flu and flu vaccines [cited 2017 Apr 13]. Available from: https://www.cdc.gov/flu/about/qa/misconceptions.htm.

6. US Agency for International Development. Pandemic influenza and other emerging threats [cited 2017 Apr 13]. Available from: https://www.usaid.gov/vietnam/pandemic-influenzaand-other-emerging-threats.

7. Ampofo WK, Azziz-Baumgartner E, Bashir U, Cox NJ, Fasce R, Giovanni $M$, et al. Strengthening the influenza vaccine virus selection and development process: report of the 3rd WHO Informal Consultation for Improving Influenza Vaccine Virus Selection held at WHO headquarters, Geneva, Switzerland, 1-3 April 2014. Vaccine 2015;33(36):4368-4382.

8. Hinh ND, Minh HV. Public health in Vietnam: scientific evidence for policy changes and interventions. Glob Health Action 2013;6:20443. 
9. Gupta V, Dawood FS, Muangchana C, Lan PT, Xeuatvongsa A, Sovann $L$, et al. Influenza vaccination guidelines and vaccine sales in southeast Asia: 2008-2011. PLoS One 2012;7(12): e52842.

10. Phan TL, Ho VT, Vu MH, Nguyen TN, Duong HT, Holt R, et al. Clinical testing of an inactivated influenza $A / H 5 N 1$ vaccine candidate in a double-blinded, placebo-controlled, randomized trial in healthy adults in Vietnam. Vaccine 2016;34(45): 5449-5456.

11. Nguyen DN, Mai le Q, Bryant JE, Hang Nle K, Hoa le NM, Nadjm $B$, et al. Epidemiology and etiology of influenza-like-illness in households in Vietnam; it's not all about the kids! J Clin Virol 2016;82:126-132.

12. Fournié G, Tripodi A, Nguyen TT, Nguyen VT, Tran TT, Bisson A, et al. Investigating poultry trade patterns to guide avian influenza surveillance and control: a case study in Vietnam. Sci Rep 2016;6:29463.

13. American College Health Association. American College Health Association-national college health assessment II: Thompson Rivers University executive summary spring 2013 [cited 2017 May 31]. Available from: https://www.tru.ca/_ shared/assets/NCHA_II_Spring_201329926.pdf.

14. Ramsey MA, Marczinski CA. College students' perceptions of H1N1 flu risk and attitudes toward vaccination. Vaccine 2011; 29(44):7599-7601.
15. Centers for Disease Control and Prevention. Key facts about seasonal flu vaccine [cited 2017 Apr 13]. Available from: https://www.cdc.gov/flu/protect/keyfacts.htm.

16. Becker H, Stuifbergen A, Oh HS, Hall S. Self-rated abilities for health practices: a health self-efficacy measure. Health Values 1993;17(5):42-50.

17. Diep PB, Tan FE, Knibbe RA, De Vries N. A multilevel study of students in Vietnam: drinking motives and drinking context as predictors of alcohol consumption. Int J Environ Res Public Health 2016;13(7).pii:E710.

18. Bui TC, Markham CM, Ross MW, Williams ML, Beasley RP, Tran $\mathrm{LT}$, et al. Perceived gender inequality, sexual communication self-efficacy, and sexual behaviour among female undergraduate students in the Mekong Delta of Vietnam. Sex Health 2012;9(4):314-322.

19. Ernsting A, Schwarzer R, Lippke S, Schneider M. 'I do not need a flu shot because I lead a healthy lifestyle': compensatory health beliefs make vaccination less likely. J Health Psychol 2013;18(6):825-836.

20. Rabiau MA, Knäuper B, Nguyen TK, Sufrategui M, Polychronakos $C$. Compensatory beliefs about glucose testing are associated with low adherence to treatment and poor metabolic control in adolescents with type 1 diabetes. Health Educ Res 2009;24(5):890-896. 